\title{
Feinberg-Horodecki Exact Momentum States of Improved Deformed Exponential-Type Potential
}

\author{
Mahmoud Farout ${ }^{1 *}$, Ahmed Bassalat ${ }^{1 \#, ~ S a m e e r ~ M . ~ I k h d a i r ~}{ }^{1,2}$ \\ ${ }^{1}$ Department of Physics, An-Najah National University, Nablus, Palestine \\ ${ }^{2}$ Department of Electrical Engineering, Near East University, Mersin, Turkey \\ Email: ^m.qaroot@najah.edu, " ahmed.bassalat@najah.edu, sameer.ikhdair@najah.edu
}

How to cite this paper: Farout, M., Bassalat, A. and Ikhdair, S.M. (2020) FeinbergHorodecki Exact Momentum States of Improved Deformed Exponential-Type Potential. Journal of Applied Mathematics and Physics, 8, 1496-1506.

https://doi.org/10.4236/jamp.2020.88115

Received: July 9, 2020

Accepted: August 11, 2020

Published: August 14, 2020

Copyright () 2020 by author(s) and Scientific Research Publishing Inc. This work is licensed under the Creative Commons Attribution International License (CC BY 4.0).

http://creativecommons.org/licenses/by/4.0/

\begin{abstract}
We obtain the quantized momentum eigenvalues, $P_{n}$, and the momentum eigenstates for the space-like Schrodinger equation, the Feinberg-Horodecki equation, with the improved deformed exponential-type potential which is constructed by temporal counterpart of the spatial form of these potentials. We also plot the variations of the improved deformed exponential-type potential with its momentum eigenvalues for few quantized states against the screening parameter.
\end{abstract}

\section{Keywords}

Quantized Momentum States, Feinberg-Horodecki Equation, The

Time-Dependent Improved Deformed Exponential-Type Potential

\section{Introduction}

In studying any physical problem in quantum mechanics we seek to find the solution of the resulting second-order differential equation. The time-dependent Schrödinger equation represents an example that describes quantum-mechanical phenomena, in which it dictates the dynamics of a quantum system. Solving this differential equation by means of any method results in the eigenvalues and eigenfunctions of that Schrödinger quantum system. However, the solution of the time-dependent Schrödinger equation analytically is exact and limited to certain problems of spatial coordinate problems [1] [2] [3] [4]. The Feinberg-Horodecki (FH) equation is an equivalent time-momentum equation to the energy-spatial coordinate Schrodinger equation which was derived by Horodecki [5] from the

${ }^{\star}$ First corresponding author.

\#Second corresponding author. 
relativistic Feinberg equation [6]. This equation has been demonstrated in a possibility of describing biological systems [7] [8] in terms of the time-like supersymmetric quantum mechanics [9]. The spatial-like solution of the FH equation can be employed to test its relevance in different areas of science including physics, biology and medicine [7] [8]. Molski obtained the spatial-like states of the time-dependent Morse oscillator potential model in the framework of the FH equation for minimizing the time-energy uncertainty relation and showed that the results are useful for interpreting the formation of the specific growth patterns during crystallization process and biological growth [7]. Furthermore, he solved the FH equation with anharmonic oscillators and obtained the space-like quantum supersymmetry for the sake of describing biological systems [8].

Recently, Bera and Sil found the exact solutions of the FH equation for the time-dependent Wei-Hua oscillator and Manning-Rosen potentials by the Nikiforov-Uvarov (NU) method [10]. A simple form of a potential model [11] named Deng-Fan oscillator potential was introduced in 1957. This potential taking a general Morse potential mesa 1998 generalized has been studied for its energy spectrum and wave functions by [11] [12] [13] [14] and related to the Manning-Rosen potential [15] [16] which is also called Eckart potential by some authors [17] [18] [19] or anharmonic potential. This system is well-defined at boundaries where $t=0$ and $t=1$. The spatial-like Deng-Fan model is quantitatively very similar to Morse model with correct asymptotic behaviour when inter nuclear separation distance comes to zero [11] and correctly describes the spectrum of diatomic molecules and electromagnetic transition [20] [21] [22]. The FH equation is solved with the time-dependent Deng-Fan oscillator potential model to obtain the exact momentum states by means of the parametric NU method [23].

Recently, Altug and Sever have studied the FH equation with time-dependent Poschl-Teller potential and found its space-like coherent states [24]. We also studied the solutions of FH equation for time-dependent mass (TDM) harmonic oscillator quantum system. An appropriate interaction to time-dependent mass is chosen to obtain the correct spectrum of stationary energy. The related spectrum of Harmonic oscillator potential acting on the TDM stationary state energies is found [25]. The exact solutions of $\mathrm{FH}$ equation under time-dependent Tietz-Wei di-atomic molecular potential have been obtained. In particular, the quantized momentum eigenvalues and corresponding wave functions are found in framework of supersymmetric quantum mechanics [26]. The spectra of general molecular potential (GMP) are obtained using asymptotic iteration method within the framework of non-relativistic quantum mechanics. The vibrational partition function is calculated in closed form and used to obtain thermodynamic functions [27].

Recently, we solved the FH equation with the time-dependent Kratzer plus screened Coulomb potential [28]; we solved FH equation with the time-dependent screened Kratzer-Hellmann potential model [29], and very recently we a general time-dependent potential [30]. In each case, we obtained the approximated eigensolutions of momentum states and wave functions by means of the NU method. 
The motivation of this work is to apply the NU method [31] for the general molecular potential having a certain time-dependence. The momentum eigenvalues, $P_{n}$, of the FH equation and the space-like coherent eigenvectors are obtained. The rest of this work is organized as follows: the NU method is briefly introduced in Section 2. The exact solution of the FH equation for the time-dependent general molecular potential is solved to obtain its quantized momentum states and eigenfunctions in Section 3. We generate the solutions of a few special potentials mainly found from our general form solution in Section 4. Finally we present our discussions and conclusions.

\section{Exact Solutions of the FH Equation for the Time-Dependent Improved Deformed Exponential-Type Potential}

The Nikiforov-Uvarov (NU) method (see Appendix) will be used to find the exact solutions of $\mathrm{FH}$ equation for the improved deformed exponential-type (IDEP) which results in momentum eigenvalues and their eigenstates.

The time-dependent of the improved deformed exponential-type potential is given by [32]

$$
V(t)=D_{e}\left[1-\frac{q-\mathrm{e}^{2 \alpha\left(t_{e}-t_{0}\right)}}{q-\mathrm{e}^{2 \alpha\left(t-t_{0}\right)}}\right]^{2} .
$$

where $t_{0}$ and $\alpha$ are adjustable real potential parameters. $q$ is a dimensionless parameter, $D_{e}$ is the dissociation energy and $t_{e}$ the equilibrium time point. The IDEP is reduces to the improved Tietz potential if $2 \alpha$ is replaced by $\alpha$ and $-q \mathrm{e}^{2 \alpha t_{0}}$ by $h$. If the IDEP potential is substituted in $\mathrm{FH}$ equation, one obtains

$$
\left[-\frac{\hbar^{2}}{2 m c^{2}} \frac{\mathrm{d}^{2}}{\mathrm{~d} t^{2}}+D_{e}\left(1-\frac{q-\mathrm{e}^{2 \alpha\left(t_{e}-t_{0}\right)}}{q-\mathrm{e}^{2 \alpha\left(t-t_{0}\right)}}\right)^{2}\right] \psi_{n}(t)=c P_{n} \psi_{n}(t) .
$$

Now, let $s=\frac{1}{q} \mathrm{e}^{2 \alpha\left(t-t_{0}\right)}$, where $s \in\left(\frac{1}{q} \mathrm{e}^{-2 \alpha t_{0}}, \infty\right)$, we get

$$
\frac{\mathrm{d}^{2} \psi_{n}(s)}{\mathrm{d} s^{2}}+\frac{1-s}{s(1-s)} \frac{\mathrm{d} \psi_{n}(s)}{\mathrm{d} s}+\frac{-\zeta_{1}^{2}-\zeta_{3} s+\zeta_{2} s^{2}}{s^{2}(1-s)^{2}} \psi_{n}(s)=0
$$

where

$$
\begin{gathered}
\beta=-\frac{2 \hbar \alpha^{2}}{m c^{2}}, \\
L=\frac{D_{e}}{\beta}, \\
M=-\frac{c P_{n}}{\beta}, \\
A=\frac{D_{e} \mathrm{e}^{4 \alpha\left(t_{e}-t_{0}\right)}}{q^{2} \beta},
\end{gathered}
$$




$$
\begin{aligned}
& C=\frac{2 D_{e} \mathrm{e}^{2 \alpha\left(t_{e}-t_{0}\right)}}{q \beta}, \\
& \zeta_{1}^{2}=-(A+M), \\
& \zeta_{2}=-(C+2 M),
\end{aligned}
$$

and

$$
\zeta_{3}=-(L+M)
$$

After comparing Equation (3) with Equation (A1), one obtains

$$
\tilde{\tau}(s)=1-s, \sigma(s)=s(1-s) \text {, and } \tilde{\sigma}(s)=-\zeta_{1}^{2}+\zeta_{2} s+-\zeta_{3} s^{2} .
$$

when these values are substituted in equation

$$
\begin{gathered}
\Pi(s)=\frac{\sigma^{\prime}-\tilde{\tau}}{2} \pm \sqrt{\left(\frac{\sigma^{\prime}-\tilde{\tau}}{2}\right)^{2}-\tilde{\sigma}+k \sigma} \text { (see NU method [28] [29] [30]), we get } \\
\Pi(s)=-\frac{s}{2} \pm \sqrt{\left(\frac{1}{4}+\zeta_{3}-k\right) s^{2}+\left(k-\zeta_{2}\right) s+\zeta_{1}^{2}}
\end{gathered}
$$

As mentioned in the NU method, the discriminant under the square root, in Equation (12), has to be zero, so that the expression of $\Pi(s)$ becomes the square root of a polynomial of the first degree. This condition can be written as

$$
\left(\frac{1}{4}+\zeta_{3}-k\right) s^{2}+\left(k-\zeta_{2}\right) s+\zeta_{1}^{2}=0
$$

After solving this equation, we get

$$
s=\frac{-\left(k+\zeta_{3}\right) \pm \sqrt{\left(k+\zeta_{3}\right)^{2}-4 \zeta_{1}^{2}\left(\frac{1}{4}-\zeta_{2}-k\right)}}{2\left(\frac{1}{4}-\zeta_{2}-k\right)} .
$$

Then, for our purpose we assume that

$$
\left(k+\zeta_{3}\right)^{2}-4 \zeta_{1}^{2}\left(\frac{1}{4}-\zeta_{2}-k\right)=0 .
$$

Arranging this equation and solving it to get an expression for $k$ which is given by the following,

$$
k_{ \pm}=\zeta_{2}-2 \zeta_{1}^{2} \pm 2 \zeta_{1}\left(\frac{1}{R}-\frac{1}{2}\right),
$$

where the expression between the parentheses is given by

$$
\frac{1}{R}-\frac{1}{2}=\sqrt{C-A-L+\frac{1}{4}} .
$$

where the parameters in this equation must be selected to let $R$ be real and the results have physical meanings. If we substitute $k_{-}$into Equation (12) we get a possible expression for $\Pi(s)$, which is given by

$$
\Pi(s)=\zeta_{1}-\left(\zeta_{1}+\frac{1}{R}\right) s,
$$


this solution satisfy the condition that the derivative of $\tau(s)$ is negative. Therefore, the expression of $\tau(s)$ which satisfies these conditions can be written as

$$
\tau(s)=1-s+2 \zeta_{1}-2 s\left(\zeta_{1}+\frac{1}{R}\right)
$$

Now, substituting the values of $\tau_{-}^{\prime}(s), \sigma^{\prime \prime}(s), \Pi_{-}^{\prime}(s)$ and $k_{-}$into Equation (A2) and Equation (A3), we obtain

$$
\lambda_{n}=\zeta_{2}-2 \zeta_{1}^{2}-2 \zeta_{1}\left(\frac{1}{R}-\frac{1}{2}\right)-\left(\frac{2 \zeta_{1}}{R}+\zeta_{1}\right)
$$

and

$$
\lambda=\lambda_{n}=n(n-1)+2 n\left(1+\frac{2}{R}\right)+2 n \zeta_{1} .
$$

Now, from Equation (20) and Equation (21), we get the eigenvalues of the quantized momentum as

$$
P_{n}=\frac{1}{c}\left(A+\beta\left[\frac{2 A-C-n(n+1)-\frac{2 n+1}{R}}{2\left(n+\frac{1}{R}\right)}\right]^{2}\right)
$$

where $\beta, A$ and $C$ are defined in Equation (4), Equation (7) and Equation (8) respectively.

Due to the NU method used in getting the eigenvalues, the polynomial solutions of the hypergeometric function $y_{n}(s)$ depend on the weight function $\rho(s)$ which can be determined by solving $\sigma(s) \rho^{\prime}(s)+[\sigma(s)-\tau(s)] \rho(s)=0$ to get

$$
\rho(s)=s^{2 \zeta_{1}}(1-s)^{\frac{2}{R}-1} \text {. }
$$

Substituting $\rho(s)$ into $y_{n}(s)=\frac{A_{n}}{\rho(s)} \frac{\mathrm{d}^{n}}{\mathrm{~d} s^{n}}\left[\sigma^{n}(s) \rho(s)\right]$, we get an expression for the wave functions as

$$
y_{n}(s)=A_{n} s^{-2 \zeta_{1}}(1-s)^{-\left(\frac{2}{R}-1\right)} \frac{\mathrm{d}^{n}}{\mathrm{~d} s^{n}}\left[s^{n+2 \zeta_{1}}(1-s)^{n+\frac{2}{R}-1}\right],
$$

where $A_{n}$ is the normalization constant. Solving Equation (24) gives the final form of the wave function in terms of the Jacobi polynomial $P_{n}^{(\alpha, \beta)}$ as follows,

$$
y_{n}(s)=A_{n} n ! P_{n}^{\left(2 \zeta_{1}, \frac{2}{R}-1\right)}(1-2 s) .
$$

Now, substituting $\Pi_{-}(s)$ and $\sigma(s)$ into $\sigma(s)=\Pi(s) \frac{\phi_{n}(s)}{\phi_{n}^{\prime}(s)}$ then solving it we obtain

$$
\phi_{n}(s)=s^{\zeta_{1}}(1-s)^{\frac{1}{R}}
$$

Substituting Equation (25) and Equation (26) in $\psi_{n}(s)=\phi_{n}(s) y_{n}(s)$, one ob- 
tains,

$$
\psi_{n}(s)=B_{n} s^{\zeta_{1}}(1-s)^{\frac{1}{R}} P_{n}^{\left(2 \zeta_{1}, \frac{2}{R}-1\right)}(1-2 s),
$$

where $B_{n}$ is the normalization constant.

\section{Numerical Results and Discussion}

We compute the momentum eigenvalues of time dependent improved deformed exponential-type potential for some diatomic molecules like $\mathrm{CO}, \mathrm{N}_{2}, \mathrm{H}_{2}$ and $\mathrm{LiH}$. This was done using the spectroscopic parameters displayed in Table 1.

Figure 1 shows the variation of the time-dependent improved deformed exponential-type potential (IDEP) well for four different diatomic molecules at small times. Hence, this potential well changes from $20 \mathrm{eV}$ to nearly $5 \mathrm{eV}$ for the diatomic molecules $\mathrm{H}_{2}$ and $\mathrm{LiH}$ whereas it changes from $50 \mathrm{eV}$ to $5 \mathrm{eV}$ for $\mathrm{N}_{2}$ diatomic molecule. However, $\mathrm{CO}$ diatomic molecule has a unique behavior; it varies from $5 \mathrm{eV}$ to $10 \mathrm{eV}$. In Figure 2, we examined the variation of the quantized momentum states $P_{n}$ of IDEP against the screening parameter $q$ for various diatomic molecules. It is seen that the momentum of the present potential model decreases monotonically from zero, for $\mathrm{H}_{2}, \mathrm{~N}_{2}$ and $\mathrm{LiH}$ whereas for $\mathrm{CO}$ it shows different behavior as it decreases from $-20 \mathrm{eV} / \mathrm{c}$, against the screening parameter $q$ for various values of states, $n$. Figure 3 shows the variation of $P_{n}$ in the field of IDEP against the exponential parameter $\alpha$ for various diatomic molecules. The diatomic molecules exhibit different features; for various values of $n$. It is obvious from figure that $P_{n}$ increases monotonically with increasing $\alpha$ for $\mathrm{CO}$

Table 1. Spectroscopic parameters of the various diatomic molecules [32].

\begin{tabular}{cccccc}
\hline Molecule & $D_{e}(\mathrm{eV})$ & $t_{e}(\mathrm{~ns})$ & $\mu$ (a.m.u $)$ & $t_{0}(\mathrm{~ns})$ & $q$ \\
\hline $\mathrm{CO}$ & 10.84514471 & 1.1283 & 6.860586000 & 1.128300118 & -0.6544806294 \\
$\mathrm{~N}_{2}$ & 9.9051 & 1.0970 & 7.0034 & 1.097000113 & -0.3543700921 \\
$\mathrm{H}_{2}$ & 4.7446 & 0.7416 & 0.5039 & 0.7416001485 & -0.3236073943 \\
$\mathrm{LiH}$ & 2.5155 & 1.5955 & 0.8801 & 1.595500403 & -0.3326882575 \\
\hline
\end{tabular}

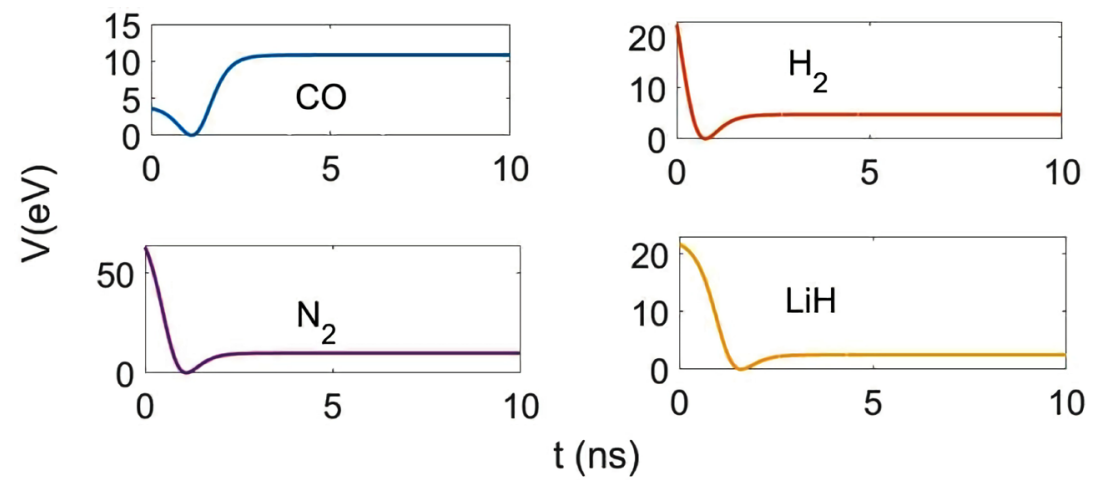

Figure 1. Improved deformed exponential-type potential (IDEP) for diatomic molecules. The parameters used are presented in Table 1 , and $\alpha=0.5(\mathrm{~ns})^{-1}$. 


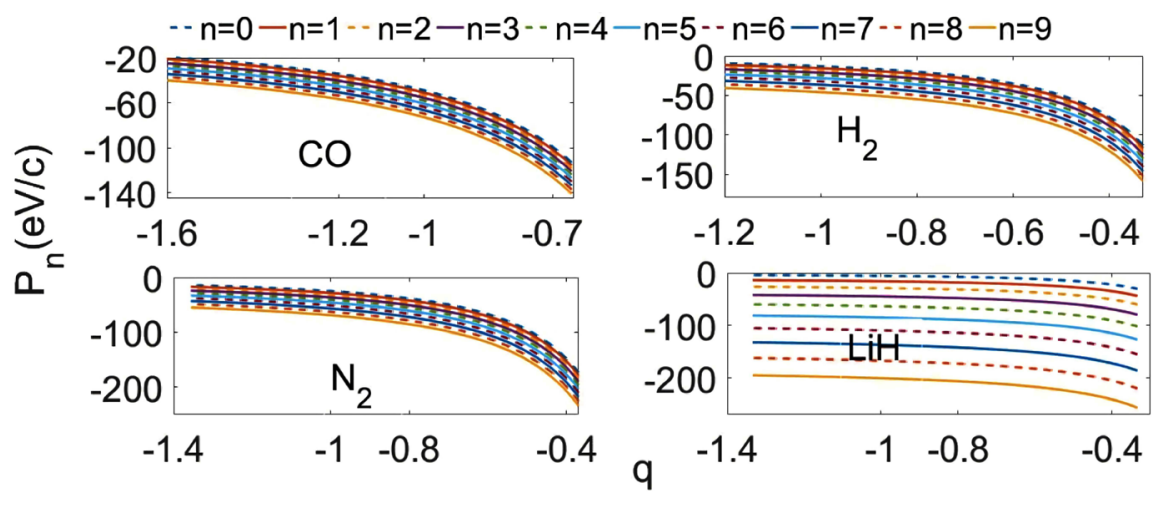

Figure 2. FH quantized momentum eigenvalues for the improved deformed exponential-type potential plotted vs $q$ for diatomic molecules. The parameters used are presented in Table 1 , and $\alpha=0.5(\mathrm{~ns})^{-1}$.

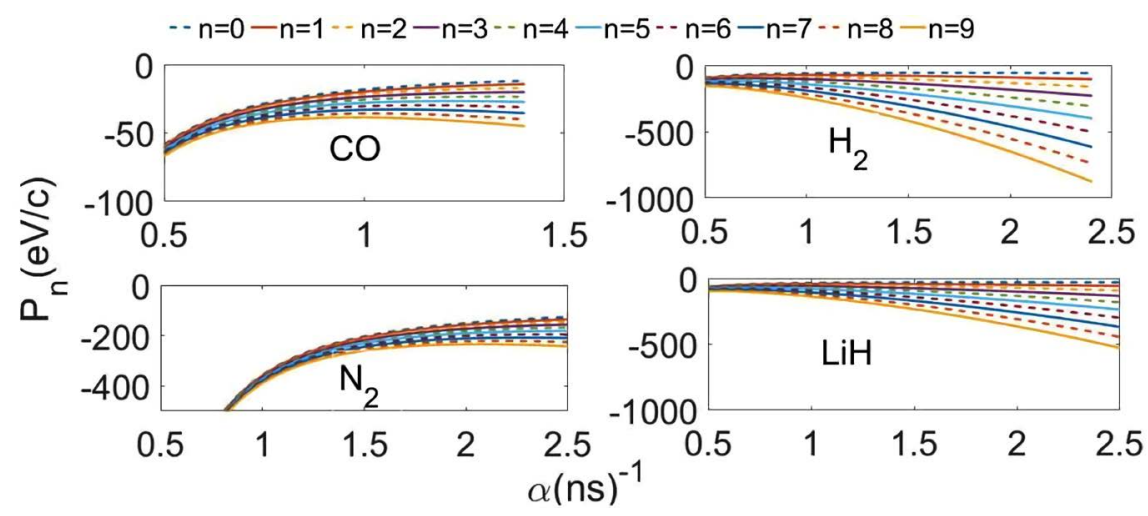

Figure 3. FH quantized momentum eigenvalues for the improved deformed exponential-type potential plotted vs $\alpha$ for diatomic molecules. The parameters used are presented in Table 1.

and $\mathrm{N}_{2}$ when particle is subjected to the aforementioned system. The reverse case happens with $\mathrm{H}_{2}$ and $\mathrm{LiH}$ diatomic molecules where $P_{n}$ decreases monotonically from a value close to zero with increasing $\alpha$.

Finally, Figure 4 shows the behavior of $P_{n}$ against state $n$ for various diatomic molecules subjected to the field of IDEP with various values of $\alpha$. It is seen that $P_{n}$ for $\mathrm{H}_{2}$ and $\mathrm{LiH}$ decreases monotonically from zero with increasing $n$. However, in the case of $\mathrm{CO}$ and $\mathrm{N}_{2}$ diatomic molecules, it is seen that $P_{n}$ decreases slightly linearly with increasing $n$.

\section{Conclusion}

We solved the Feinberg-Horodecki (FH) equation for the time-dependent improved deformed exponential-type potential via Nikiforov-Uvarov (NU) method. We got the exact quantized momentum eigenvalues solution of the $\mathrm{FH}$ equation. It is therefore, worth mentioning that the method is elegant and powerful. Our results can be applied in biophysics and other branches of physics. We find that our analytical results are in good agreement with other findings in literature. We have shown the behaviors of the improved deformed exponential-type potential 


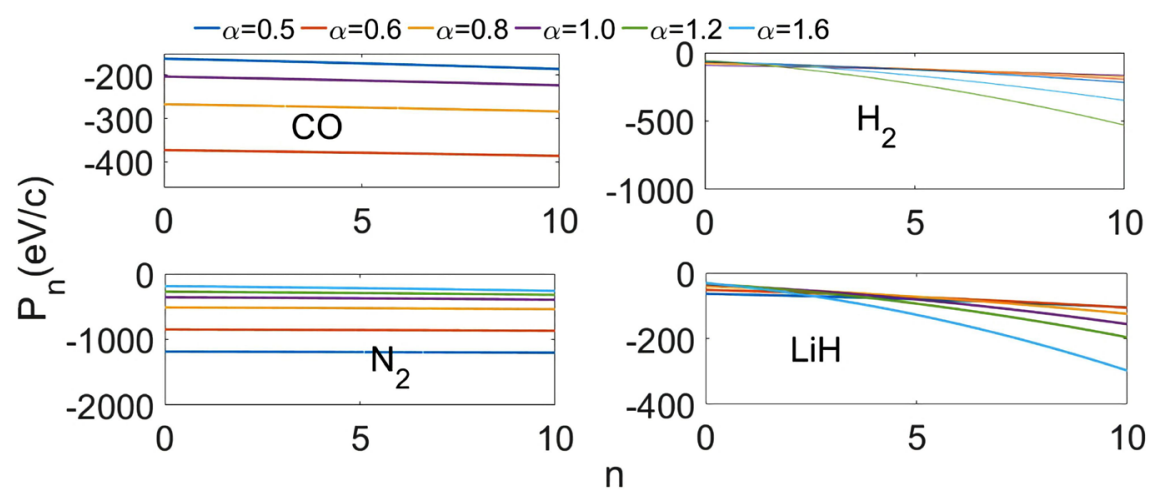

Figure 4. FH quantized momentum eigenvalues for the improved deformed exponential-type potential plotted vs $n$ for diatomic molecules. The parameters used are presented in Table 1.

against screening parameters. Further, taking spectroscopic values for the potential parameters, we plotted the quantized momentum of few states against the screening parameter for diatomic molecules. Our results are good agreements with the energy bound states.

\section{Acknowledgements}

We thank the Editor and the referees for their valuable comments. This research is funded by Winter School in High Energy Physics in Palestine (WISHEPP). This generous support is greatly appreciated.

\section{Conflicts of Interest}

The authors declare no conflicts of interest regarding the publication of this paper.

\section{References}

[1] Park, T.J. (2002) Exactly Solvable Time-Dependent Problems: Potentials of Monotonously Decreasing Function of Time. Bulletin of the Korean Chemical Society, 23, 1733-1736. https://doi.org/10.5012/bkcs.2002.23.12.1733

[2] Vorobeichik, I., Lefebvre, R. and Moiseyev, N. (1998) Field-Induced Barrier Transparency. Europhysics Letters, 41, 111-116. https://doi.org/10.1209/epl/i1998-00117-6

[3] Shen, J.Q. (2003) Solutions of the Schrödinger Equation for the Time-Dependent Linear Potential.

[4] Feng, M. (2001) Complete Solution of the Schrödinger Equation for the Time-Dependent Linear Potential. Physical Review A, 64, Article ID: 034101. https://doi.org/10.1103/PhysRevA.64.034101

[5] Horodecki, R. (1988) Inference for a Hazard Rate Change Point. Il Nuovo Cimento $B$ (1971-1996), 102, 27-32. https://doi.org/10.1007/BF02728791

[6] Feinberg, G. (1967) On Testing for a Constant Hazard against a Change-Point Alternative. Physical Review, 159, 1089-1105. https://doi.org/10.1103/PhysRev.159.1089

[7] Molski, M. (2006) Space-Like Coherent States of Time-Dependent Morse Oscillator. 
The European Physical Journal D Atomic, Molecular, Optical and Plasma Physics, 40, 411-416. https://doi.org/10.1140/epjd/e2006-00182-3

[8] Molski, M. (2010) Biosupersymmetry. Biosystems, 100, 47-54. https://doi.org/10.1016/j.biosystems.2010.01.001

[9] Witten, E. (1981) Dynamical Breaking of Supersymmetry. Nuclear Physics B, 188, 513-554. https://doi.org/10.1016/0550-3213(81)90006-7

[10] Bera, P.K. and Sil, T. (2013) Exact Solutions of Feinberg-Horodecki Equation for Time-Dependent Anharmonic Oscillator. Pramana, 80, 31-39. https://doi.org/10.1007/s12043-012-0358-6

[11] Deng, Z.H. and Fan, Y.P. (1957) A Potential Function of Diatomic Molecules. Journal of Shandong University (Natural Science), 1. http://en.cnki.com.cn/Article_en/CJFDTotal-SDDX195701011.htm

[12] Wang, P.Q., Zhang, L.H., Jia, C.S. and Liu, J.Y. (2012) Equivalence of the Three Empirical Potential Energy Models for Diatomic Molecules. Journal of Molecular Spectroscopy, 274, 5-8. https://doi.org/10.1016/j.jms.2012.03.005

[13] Rong, Z., Kjaergaard, H.G. and Sage, M.L. (2003) Comparison of the Morse and Deng-Fan Potentials for XH Bonds in Small Molecules. Molecular Physics, 101, 2285-2294. https://doi.org/10.1080/0026897031000137706

[14] Mesa, A.D.S., Quesne, C. and Smirnov, Y.F. (1998) Generalized Morse Potential: Symmetry and Satellite Potentials. Journal of Physics A: Mathematical and General, 31, 321. https://doi.org/10.1088/0305-4470/31/1/028

[15] Infeld, L. and Hull, T.E. (1951) The Factorization Method. Reviews of Modern Physics, 23, 21. https://doi.org/10.1103/RevModPhys.23.21

[16] Manning, M.F. and Rosen, N. (1933) A Potential Function for the Vibrations of Diatomic Molecules. Physical Review, 44, 951-954.

[17] Dabrowska, J.W., Khare, A. and Sukhatme, U.P. (1988) Explicit Wave Functions for Shape-Invariant Potentials by Operator Techniques. Journal of Physics A: Mathematical and General, 21, L195. https://doi.org/10.1088/0305-4470/21/4/002

[18] Cooper, F., Khare, A. and Sukhatme, U. (1995) Supersymmetry and Quantum Mechanics. Physics Reports, 251, 267-385.

https://doi.org/10.1016/0370-1573(94)00080-M

[19] Zhang, X.C., Liu, Q.W., Jia, C.S. and Wang, L.Z. (2005) Bound States of the Dirac Equation with Vector and Scalar Scarf-Type Potentials. Physics Letters A, 340, 59-69. https://doi.org/10.1016/j.physleta.2005.04.011

[20] Dong, S.H. and Gu, X.Y. (2008) Arbitrary L State Solutions of the Schrödinger Equation with the Deng-Fan Molecular Potential. Journal of Physics. Conference Series, 96, Article ID: 012109. https://doi.org/10.1088/1742-6596/96/1/012109

[21] Hamzavi, M., Ikhdair, S.M. and Thylwe, K.E. (2013) Equivalence of the Empirical Shifted Deng-Fan Oscillator Potential for Diatomic Molecules. Journal of Mathematical Chemistry, 51, 227-238. https://doi.org/10.1007/s10910-012-0075-x

[22] Oluwadare, O.J., Oyewumi, K.J., Akoshile, C.O. and Babalola, O.A. (2012) Approximate Analytical Solutions of the Relativistic Equations with the Deng-Fan Molecular Potential Including a Pekeris-Type Approximation to the (Pseudo or) Centrifugal Term. Physica Scripta, 86, Article ID: 035002. https://doi.org/10.1088/0031-8949/86/03/035002

[23] Hamzavi, M., Ikhdair, S.M. and Amirfakhrian, M. (2013) Exact Solutions of Feinberg-Horodecki Equation for Time-Dependent Deng-Fan Molecular Potential. Theoretical and Applied Physics, 7, 40-43. https://doi.org/10.1186/2251-7235-7-40 
[24] Arda, A. and Sever, R. (2017) Feinberg-Horodecki Equation with Pöschl-Teller Potential: Space-Like Coherent States. Zeitschrift fr Naturforschung A, 72, 541-545. https://doi.org/10.1515/zna-2017-0053

[25] Eshghi, M., Sever, R. and Ikhdair, S.M. (2016) Exact Analytical Approach to Differential Equations with Variable Coefficients. The European Physical Journal Plus, 131, 223-229. https://doi.org/10.1140/epjp/i2016-16386-9

[26] Ojonubah, J.O. and Onate, C.A. (2016) Exact Solutions of Feinberg-Horodecki Equation for Time-Dependent Tietz-Wei Diatomic Molecular Potential. The African Review of Physics, 10, 453-456.

[27] Ikot, A.N., Chukwuocha, E.O., Onyeaju, M.C., Onate, C.A., Ita, B.I. and Udoh, M.E. (2018) Thermodynamics Properties of Diatomic Molecules with General Molecular Potential. Pramana, 90, 22. https://doi.org/10.1007/s12043-017-1510-0

[28] Farout, M., Sever, R. and Ikhdair, S.M. (2020) Approximate Solution to the Time-Dependent Kratzer plus Screened Coulomb Potential in the Feinberg-Horodecki Equation. Chinese Physics B, 29, Article ID: 060303.

https://doi.org/10.1088/1674-1056/ab8379

[29] Farout, M. and Ikhdair, S.M. (2020) Momentum Eigensolutions of Feinberg-Horodecki Equation with Time-Dependent Screened Kratzer-Hellmann Potential. Journal of Applied Mathematics and Physics, 8, 1207-1221. https://doi.org/10.4236/jamp.2020.87091

[30] Farout, M., Bassalat, A. and Ikhdair, S.M. (2020) Exact Quantized Momentum Eigenvalues and Eigenstates of a General Molecular Potential Model. Journal of Applied Mathematics and Physics, 8, 1434-1447. https://doi.org/10.4236/jamp.2020.87109

[31] Nikiforov, A.F. and Uvarov, V.B. (1988) Special Functions of Mathematical Physics. Vol. 205, Birkhäuser, Basel. https://doi.org/10.1007/978-1-4757-1595-8

[32] Okorie, U., Ikot, A., Chukwuocha, E.O. and Rampho, G. (2020) Thermodynamic Properties of Improved Deformed Exponential-Type Potential (idep) for Some Diatomic Molecules. Journal of Mathematical Chemistry, 58, 989-1013. https://doi.org/10.1016/j.rinp.2020.103078 


\section{Appendix: Methodology}

The Nikiforov-Uvarov (NU) [31] method is an efficient technique usually employed to reduce the second-order differential equation into a general form of a hypergeometric type equation. Therefore, any second order differential equation can be transformed, via an appropriate coordinate transformation $s=s(t)$, into a standard form:

$$
\psi_{n}^{\prime \prime}(s)+\frac{\tilde{\tau}(s)}{\sigma(s)} \psi_{n}^{\prime}(s)+\frac{\tilde{\sigma}(s)}{\sigma^{2}(s)} \psi_{n}(s)=0,
$$

where $\sigma(s)$ and $\tilde{\sigma}(s)$ are polynomials, at most second-order, and $\tilde{\tau}(s)$ is of a first-order polynomial. To follow the method in details the reader is advised to follow [28] [29] [30] [31]. The eigenvalues equation can be found simply by solving the Equation (A2) and Equation (A3). Where

$$
\lambda=\lambda_{n}=-n \tau^{\prime}(s)-\frac{n(n-1)}{2} \sigma^{\prime \prime}(s),
$$

and

$$
\lambda=\lambda_{n}=k+\Pi^{\prime}(s)
$$

with $n=0,1,2, \cdots$, and $\tau(s)$ is a polynomial with a negative first derivative to generate an appropriate solution for the hypergeometric equation. And Further, $\Pi(s)$ is a polynomial which depends on the transformation function $s(t)$ and $k$ should be determined to calculate $\Pi(s)$, for which the discriminant under the square root is set to zero, in order to let $\Pi(s)$ to be a first order polynomial. 